\title{
Fast and Efficient Evaluation of Building Damage from Very High Resolution Optical Satellite Images
}

\author{
David Dubois, Graduate student member, IEEE, Richard Lepage, Member, IEEE
}

\begin{abstract}
In this paper we present a novel combination of object features to both match buildings from pre-disaster images to shapes in a post-disaster image and assess damage on those buildings. These features include scale profile ratios extracted from a tree of shapes representation of the original image as well as texture features. A supervised classifier is used to classify building damage into three representative classes tied to the European Macroseismic Scale (EMS-98). The method is compared to visual inspection results as well as other automated methods. Results clearly show the benefits of our method for fast crisis mapping applications with few human inputs required.
\end{abstract}

Index Terms-Disaster response, Image classification, Image processing, Remote sensing, Texture analysis

\section{INTRODUCTION}

$\mathrm{T}$ HE launch of multiple spaceborne imaging sensors in the past ten years has led to an increase in the use of remotely sensed images and research activities for remote sensing applications. Using Earth observation sensors for emergency response when disasters occur is one of the usages that are gaining more trust. Many disasters occur each year and response time is always a crucial factor. In order to adequately provide emergency relief, rescuers need to have a global picture of the situation on-site. Remote sensing can provide this kind of information.

One reason the use of remote sensing for emergency response has grown considerably in recent years is the creation and continuous support of the International Charter "Space and Major Disasters" in 2000 [1]. The purpose of the Charter is to provide a unique system for acquiring raw data, preparing useful Earth observation products and delivering them to decision makers and rescue organizations when a major disaster strikes. There are protocols defined for each kind of disaster so that adequate sensors are used with proper parameters to facilitate analysis. However, being driven by

Manuscript received March $15^{\text {th }} 2013$. First revision received February $27^{\text {th }}$ 2014 and second revision received $16^{\text {th }}$ June 2014. Manuscript accepted June $20^{\text {th }}$ 2014. This work was supported in part by the Natural Sciences and Engineering Research Council of Canada, the Fonds de recherche Nature et technologies of Quebec and Effigis Geo-Solutions Inc.

D. Dubois is with Ecole de technologie superieure, Montreal, QC H3C 1K3 Canada (e-mail: ddubois@livia.etsmtl.ca).

R. Lepage is with Ecole de technologie superieure, Montreal, QC H3C 1K3 Canada (e-mail: richard.lepage@etsmtl.ca). "best try" policies, it is sometimes impossible to get all the necessary data rapidly after the event.

Our main objective is to propose a semi-automated method for damage assessment using few resources and in a short time. To achieve this goal, we use a level set based transformation to model the image as a hierarchical representation of shapes from which meaningful shapes are extracted. This process necessitates the generation of various scale and geometric features that are then combined in a novel way to form feature vectors for supervised learning to evaluate damages. Originality of this work is in the use of specific scale features and textural features for damage assessment from preand post-event images. All of this is performed in an objectoriented manner to reduce processing time and memory usage.

The paper is divided into four main sections. The first section presents a literature review on the state of the art in building damage evaluation from VHR satellite images. The second section encompasses our proposed method for fast semi-automated damage estimation. The third section is composed of experimentations and results using the proposed method. Finally, a conclusion is made on the usefulness of the proposed method for disaster response through the Charter.

\section{LITERATURE REVIEW}

For our review, we analyzed papers directly related to buildings damage estimation.

We note three main themes for damage evaluation work: visual analysis only, hybrid method using both visual analysis and automated processes and completely automated methods. The advantages and inconveniences associated with each type of process will be presented.

\section{A. Damage evaluation}

Earth observation data is also solicited to evaluate damages caused by disaster events [2-6]. As for building detection, optical and SAR data are either used separately or combined to achieve the goal of estimating damage. In [2], the authors present a method based on the newly developed pictometry method. Although high classification accuracy can be obtained and information on building facades can be extracted, the method requires five VHR images acquired at precise angles over the scene. In [7], the authors test a correlation metric with a supervised classifier on various optical VHR datasets with 
promising results. However, the buildings are manually extracted which is what we aim to avoid. The method is simple to implement but speed performances need to be improved for disaster response. In [8], the authors propose a building damage evaluation method based on One-Class Support Vector Machine (OCSVM). A comparison is made between pixel-based and object-based approaches with clearly superior accuracy from the latter. Results are in the same range of accuracy as those from [7] but use only two classes (damaged and undamaged) instead of three or four classes to get more details on the damage levels. Building footprints are obtained through a watershed segmentation algorithm using empirical thresholds and without presenting the evaluation of the accuracy of the segmentation. In [9], automated change detection is proposed based on isotropic frequency filtering, spectral and texture analysis, and segmentation. The proposed method yields an overall accuracy of $80 \%$, better than standard change detection algorithms. Texture is computed through the homogeneity and energy features of the GLCM (Gray Level Co-occurrence Matrix), a very time consuming process.

Many researchers have proposed the use of SAR post-event images to detect damage because SAR image acquired quickly after the event can be used even if clouds are covering the area or if the image is acquired during nighttime. In [10], TerraSAR-X post-event images are used to detect building damage after the 2008 Wenchuan Earthquake. Very precise SAR models of damaged buildings are generated through simulation of the scene in order to visually estimate damage from the acquired images. Only qualitative results are presented so it is difficult to assess the full potential of SAR imagery from this paper. By using pre- and post-event SAR images in [11], the authors use a change detection approach using the backscattering coefficient and a correlation coefficient to separate damage in seven classes. The method did not include a quantitative study and the authors only make a brief comment on the necessity of acquiring pre- and postevent images with identical parameters and if possible in similar seasonal conditions to avoid backscattering and correlation differences not attributable to damage. In [12], the authors propose an extension of their previous work on SARbased seismic damage assessment. Comparison between a damaged area ratio and homogeneity and entropy features extracted from the computation of the GLCM provides good results for VHR SAR produced by COSMO-SkyMed, but this resource is scarce and is not acquired as a priority when a disaster strikes. The authors extend the experimentation with lower resolution SAR data and find good correlation between homogeneity feature and damage. GLCM computation is still time consuming and damage estimation is only two levels: undamaged or hardly damaged.

As for using optical and SAR images, a usual method is to use an optical VHR image to detect buildings and a SAR image to evaluate damage [13]. The proposed method facilitates the damage evaluation no matter the weather conditions after the disaster but requires precise registration between the different images to insure correct evaluation.
It is important to note that most operational agencies still use visual inspection to achieve acceptable damage estimation $[4,6]$. Visual inspection takes time, expert knowledge and many analysts to cover the area affected by a disaster. Automated evaluation methods are still not completely integrated into operational image processing chains.

\section{B. Pros and cons of current methods}

As can be seen from the literature, there are many different methods proposed to automate the task of evaluating building damages from disaster images. A still quite popular method consists of inspecting visually the images and estimating building extents and damage ratios in a manual fashion. Although this activity is usually done by experts, it is still prone to the subjectivity of the analyst and human error. Supervised classification scheme also depend on the training labels assigned by the operator and thus only an experienced user should be tasked with this. Some supervised methods, like SVM, can allow training errors. We also demonstrate in the result section that some features help to reduce the impact of training samples selection. Automated methods avoid some of these problems while getting high accuracy, but they usually depend on experimental thresholds that are difficult to set or need specific image acquisition modes and contextual information. Another problem with the usual segmentation and classification methods is that they have difficulties when buildings are too close to each other, which is usually the case of dense cities and shanty towns.

From our observations we have determined key components of a successful building damage evaluation scheme and propose a complete method in the following section.

\section{PROPOSED METHOD}

In this section, we present our semi-automatic method for detecting buildings and estimating their level of destruction from pre- and post-event VHR satellite imagery. The work presented in this article is the continuity of our previous work on fast building extraction [14]. The whole process is summarized in Fig. 1. For this paper, we only present the results for the damage evaluation part. To independently assess the damage evaluation capacity of our method, all the shapes defined as buildings according to the vector data layer from the Atlas of building damage assessment are used.

The three main steps in our damage evaluation algorithm are described in detail in this section: Shape matching (See Fig. 3), Texture extraction and shape difference computation (See Fig. 4) and classification (See Fig. 5). First, we present the image representation, segmentation and feature extraction scheme applied in our previous work on building classification as it is also used in this work. We used subscripts bef and aft to represent objects in images acquired either before or after the disaster respectively. The $\tau$ subscript is used to represent an algorithm or process applied to images or objects irrespective of its position in time.

Because our aim is to provide a fast method to help photointerpreters when an earthquake occurs and the Charter is 


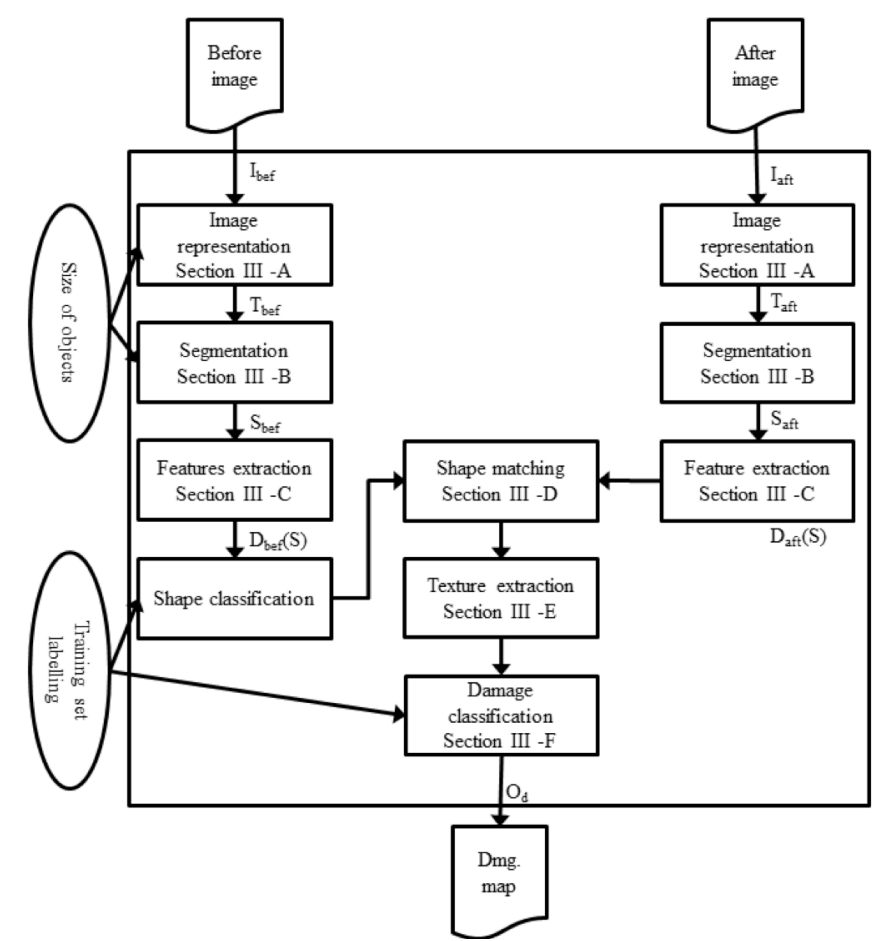

Fig. 1. Process diagram for the proposed building extraction and damage evaluation method.

activated, some limits need to be defined. The Emergency onCall Officer has operational guidelines [15] for selecting useful sensors and for setting proper acquisition parameters. Using these guidelines ensures a minimum amount of standardization that should lower generalization issues. Second, we limit ourselves to use one pre-event image and one post-event image. Third, the method should have the fewest operator-controlled parameters and these should be related to physical measures. This will avoid the creation of a black-box method where the operator shifts the parameters randomly until an adequate result is achieved.

\section{A. Fast Level Set Transform}

The Fast Level Set Transform (FLST) was first proposed in [16]. This transform enables the representation of an image and its objects as an inclusion tree. The root of that tree is a shape that covers the whole image while the end nodes are the smallest possible shapes associated with each pixel from the image. Levels in between are inclusion shapes forming a hierarchical shape structure. The FLST is based on a simple region growing algorithm. The image is scanned pixel by pixel in sequence. When a local intensity extremum is found, adjacent pixels are explored to grow a region of interest. If this region is found to be a regional extremum (having an intensity value either globally lower or higher than all neighboring regions), it is tagged as a probable shape and it is removed from the image. The region growing process then continues creating a possible parent for the extracted shape. Considering both high and low intensity regions at the same time enables the creation of a single tree structure as opposed to a pair of trees generated by applying morphological operators on bright and dark objects [17]. Once the whole image has been scanned a single time, the inclusion tree of the image is complete. The process is fast, making use of queues with heap sorting to store and retrieve neighboring pixels to compare them with the current pixel. This algorithm only changes the representation of the information contained in the image. Further processing is thus needed to extract features. An example of the FLST applied to an image is shown in Fig. 2. In this case the image generates a tree with 127 levels. Starting at the root (level 0) each level contains nested shapes obtained from the region growing process.

\section{B. Scale mapping}

Various authors have proposed the extraction of either a local or a global measure of object scale for images [18-20]. This measure can be used to index images and retrieve similar ones [21] or to help select the proper multiscale pyramidal level to detect particular objects. Most local scale mapping methods are either too sensitive to noise or are dependent on a large sliding window that results in low resolution scale 


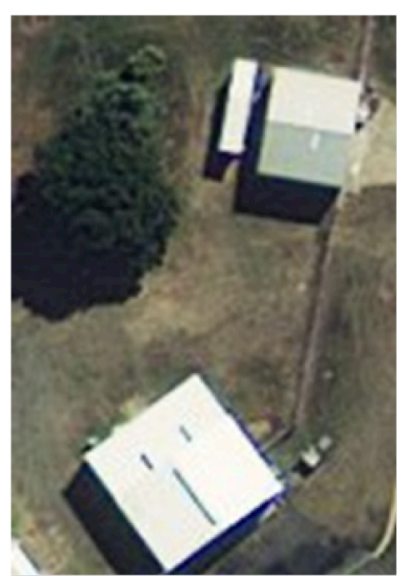

(a)

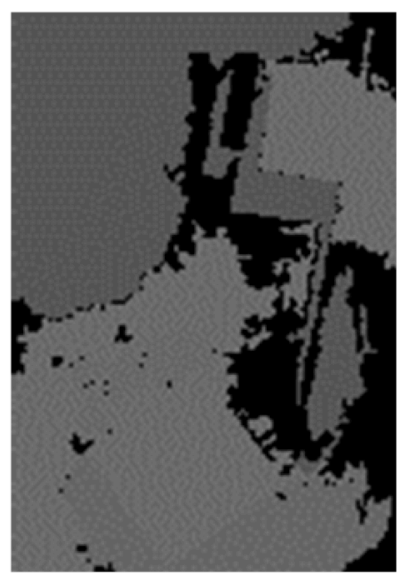

(c)

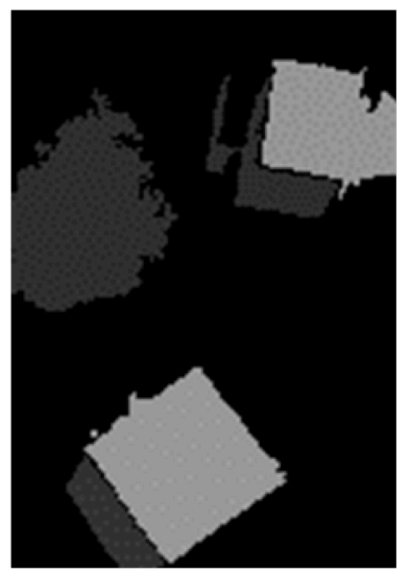

(e)

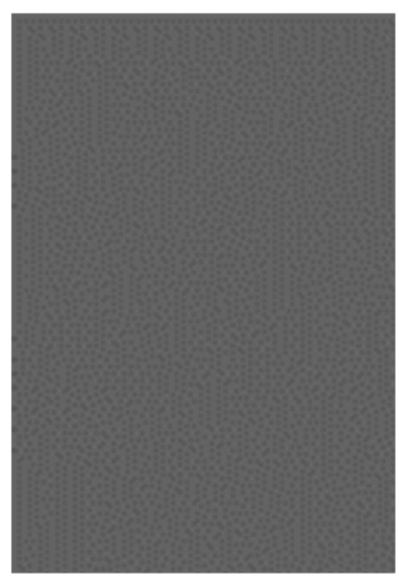

(b)

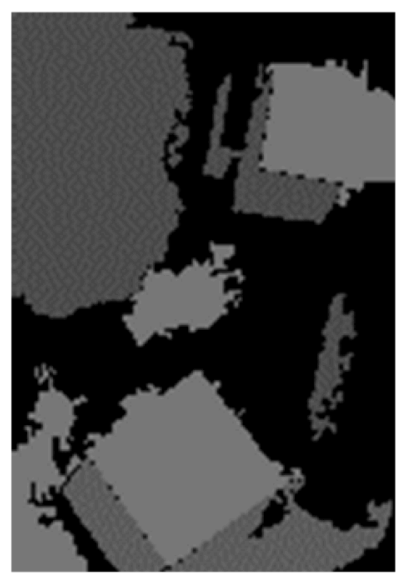

(d)

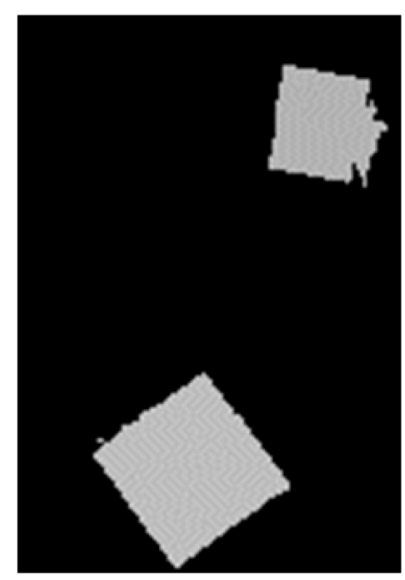

(f)
Fig. 2. FLST example. Original image (a), root of the tree of shapes (b), level 10 (c), level 20 (d), level 50 (e) and level 80 (f).

information. The method proposed in [22], called cartographic scale mapping, is based on the topography of the image for precise scale identification as well as adaptive filtering to attenuate sensitivity to noise. The interesting point about this method is not only the extraction of a "best scale" for each pixel, but also the selection of a unique relevant shape for each of those pixels. We thus use the scale mapping algorithm to extract relevant shapes that will become candidate buildings.

The method works by accumulating contrast from similar shapes in the hierarchy for a given pixel $p$. Contrast $C\left(s_{i}\right)$ of shape $s_{i}$ is computed by the absolute difference of grey intensity values $I$ between the current shape and the child shape $s_{i-1}$ on the same branch. This is noted as

$C\left(s_{i}\right)=\left|I\left(s_{i}\right)-I\left(s_{i-1}\right)\right|$.

The similarity criteria defined in equation (2) is based on the variation of area from one shape to the next by a certain fraction of the perimeter controlled by a $\lambda$ parameter which is usually set to 1 for usual images (it represents the blur width of contours for objects), as in

$\overline{\mathrm{C}}\left(s_{i}\right)=\sum_{\mathrm{k}=\mathrm{a}(\mathrm{i})}^{\mathrm{i}} \mathrm{C}\left(s_{k}\right)$.

Where for all $i$ :

$\mathrm{a}(\mathrm{i})=\min \left\{\begin{array}{c}\mathrm{j} \mid \forall \mathrm{k}=\mathrm{j}+1, \ldots, \mathrm{i}, \\ \mathrm{A}\left(s_{k}\right)-\mathrm{A}\left(s_{k-1}\right) \leq \lambda \mathrm{P}\left(s_{k-1}\right)\end{array}\right\}$.

Where $\bar{C}\left(s_{i}\right)$ is the cumulative contrast for shapes $s_{i}$ of pixel $p$ while $A$ and $P$ are the area and perimeter of a particular shape. Shape regularity can also be taken into account so that less regular shape can be discarded (this can remove undesirable transitional shapes caused by the segmentation process). The $\gamma_{s}$ parameter is used for this. Setting it to zero will avoid taking into account the regularity of shapes while any other positive value will allow increasing degrees of regularity as shown in equation (4) where the new contrast value $T$ is defined.

$\mathrm{T}\left(s_{i}\right)=\overline{\mathrm{C}}\left(s_{i}\right) \times\left(\frac{\mathrm{A}\left(s_{i}\right)}{\mathrm{P}\left(\mathrm{s}_{\mathrm{i}}\right)^{2}}\right)^{\gamma_{\mathrm{s}}}$

$\hat{S}_{c}(p)=\underset{i \in N}{\operatorname{sargmax}}\left(T\left(S_{i}\right)\right)$

The most contrasted shape $\hat{s}_{c}(p)$ is thus selected and when each pixel has been assigned to a shape, the segmentation is over. In the event that multiple candidates have the same cumulated contrast value, the one with the lowest scale is selected thus favoring buildings over city blocks. Each shape can then be represented by its scale which is the ratio area/perimeter.

\section{Feature extraction}

Buildings' damage evaluation is achieved through a supervised classification using features extracted from the scale shapes. The features are: shape scale, area and perimeter, invariant centered moments-based features, shape scale profile points and texture features. The centered moments are obtained by: 
$\mu_{k l}=\sum\left(x-\frac{m_{10}}{m_{00}}\right)^{k}\left(y-\frac{m_{01}}{m_{00}}\right)^{l}$

Where $\mu_{k l}$ is the centered moment for order $k l$ and $m_{00}, m_{l 0}$, $m_{01}$ are the area of the shape, the first order moment in the $\mathrm{x}$ axis and the first order moment in the y axis respectively. We use $\mu_{11}, \mu_{20}$ and $\mu_{02}$ to create the inertia matrix $\boldsymbol{I}_{\boldsymbol{s}}$ of the shape to get two rotation and translation invariant features. We also use $\mu_{21}, \mu_{12}, \mu_{30}$ and $\mu_{03}$ to get two more features:

$I_{s}=\left[\begin{array}{ll}\mu_{20} & \mu_{11} \\ \mu_{11} & \mu_{02}\end{array}\right]$

$r_{1}=m_{00}$

$r_{2}=\mu_{20}+\mu_{02}=\operatorname{tra}\left(I_{s}\right)$

$r_{3}=\mu_{20} * \mu_{02}-\mu_{11} * \mu_{11}=\operatorname{det}\left(I_{s}\right)$

$r_{4}=\left(\mu_{30}-3 \mu_{12}\right)^{2}+\left(\mu_{03}-3 \mu_{21}\right)^{2}$

$r_{5}=\left(\mu_{30}+\mu_{12}\right)^{2}+\left(\mu_{03}+\mu_{21}\right)^{2}$

$r_{l}$ is the area of the shape and this feature will also be used for shape matching later on. For the scale features, we recall (2) in which contrast is cumulated for shapes. The various pixels will have different levels in the tree of shapes depending on regional variations but from our experience pixels associated with buildings generally have at least five cumulated contrast shapes present in the tree. Taking the contrast value weighted by the scale (area/perimeter) for these five potential shapes (using (2)) for a pixel will yield five features for the selected shape. The details of extraction and usefulness of these features are presented in [23].

\section{Shape matching}

In order to estimate damage on existing buildings only, we must first match shapes in the post-event image with extracted buildings from the pre-event image. With the precise orbital information from the newest satellites and latest registration methods, registration of images should be considered as adequate. In the case where the images cannot be precisely registered, we present a method for matching shapes when only coarse registration of the two images is available. An image registration algorithm based on shapes is proposed in [24] but our tests lead to poor offsets selection making the registration unusable. We believe this is caused by the lack of enough undamaged buildings in the post-event image. Since there is no guarantee there will be a sufficient number of highly corresponding pairs from the pre- and post-event images, we suggest using a simple similarity matching algorithm to get a coarse (less than 10 pixels error) registration. The process diagram for this algorithm is presented in Fig. 3.

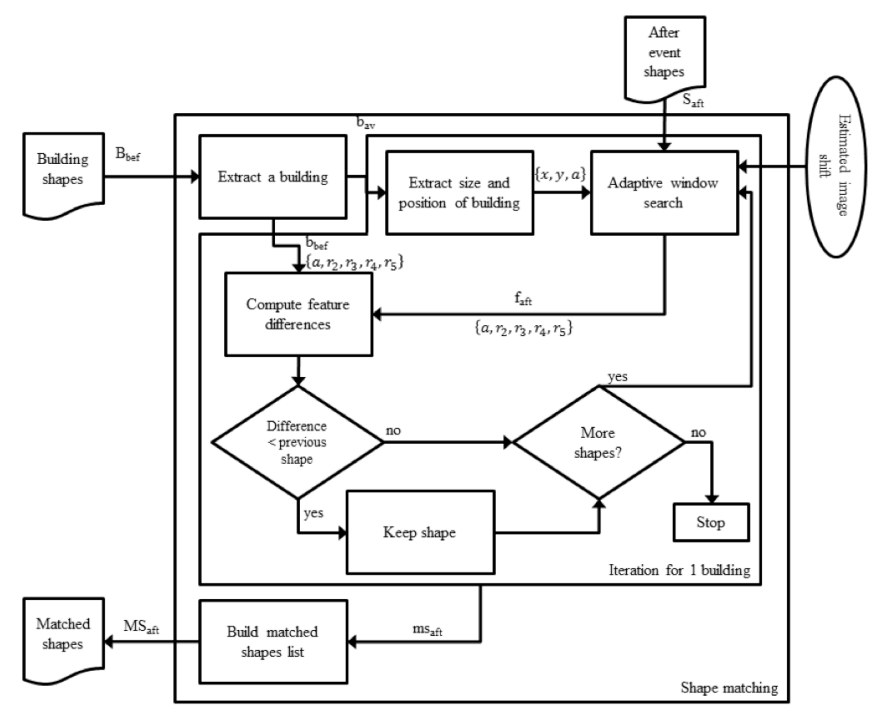

Fig. 3. Process diagram for the proposed shape matching method.

From this point, shapes detected as buildings in the pre-event image can be matched to shapes in the post-event image. To achieve this, the already extracted rotation and translation invariant features $r_{1}, r_{2}$ and $r_{3}$ are used. For each building detected, the shape from the post-event image whose centroid is within a certain distance from the centroid of the building with the most similar features is considered to be the matching shape. We set the search window by calculating the length of the side of a square having the same area as the building while the similarity is computed by minimizing feature differences according to (13).

$\min C\left(b_{b e f k}, f_{a f t f}\right)=\sqrt{\sum_{i=1}^{n}\left(r_{b e f k i}-r_{a f t f i}\right)^{2}}$

\section{E. Texture extraction}

Multiple features can be used to differentiate buildings that are moderately damaged or intact, those that are more damaged and those that are badly damaged or completely destroyed. The shape matching procedure can provide some cues about the level of destruction. The distance between preevent and post-event centroids for shape pairs and the difference between invariant features should be linked to the amount of destruction (i.e.: displaced centroids can indicate shifted rooftop). The changes in texture features from pre- and post-event images can also provide information on the state of buildings. In Fig. 4, we describe the process of extracting texture features from pre-event buildings and post-event matched shapes as well as the creation of shapes based on feature differences between the buildings extracted before and 
the shapes extracted after the earthquake.

Textural information can provide hints about changes taking place between the two acquisition dates. Laws [25] proposed a set of energy measurements to determine the texture properties of a region of the image. These measurements are based on the average grey level (L), edges (E), spots (S), ripples (R) and waves (W). To obtain $5 \times 5$ 2D kernels, the outer product of all combinations of the five 1D kernels in (14) is computed (see (15) for an example of a $\mathbf{E 5 L 5}$ kernel from $E 5^{T} \times L 5$ ).

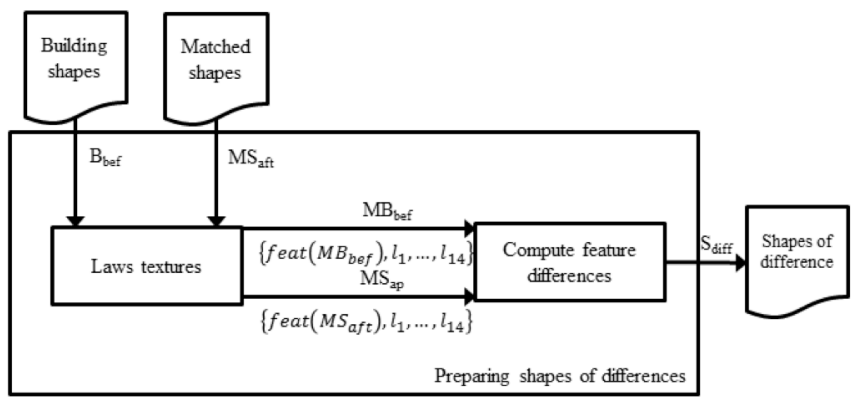

Fig. 4. Process diagram for extracting texture and computing differences. [2 columns wide!!!]

$$
\begin{aligned}
L 5 & =\left[\begin{array}{llllr}
1 & 4 & 6 & 4 & 1
\end{array}\right] \\
E 5 & =\left[\begin{array}{llllr}
-1 & -2 & 0 & 2 & 1
\end{array}\right] \\
S 5 & =\left[\begin{array}{llllr}
-1 & 0 & 2 & 0 & -1
\end{array}\right] \\
R 5 & =\left[\begin{array}{lllll}
1 & -4 & 6 & -4 & 1
\end{array}\right] \\
W 5 & =\left[\begin{array}{llllr}
-1 & 2 & 0 & -2 & 1
\end{array}\right]
\end{aligned}
$$

$$
\begin{aligned}
& {\left[\begin{array}{c}
-1 \\
-2 \\
0 \\
2 \\
1
\end{array}\right] \times\left[\begin{array}{ccccc}
1 & 4 & 6 & 4 & 1
\end{array}\right]=} \\
& {\left[\begin{array}{ccccc}
-1 & -4 & -6 & -4 & -1 \\
-2 & -8 & -12 & -8 & -2 \\
0 & 0 & 0 & 0 & 0 \\
2 & 8 & 12 & 8 & 2 \\
1 & 4 & 6 & 4 & 1
\end{array}\right]=E 5 L 5}
\end{aligned}
$$

This yields 25 convolution kernels that are each applied to the original image resulting in 25 filtered images. It is then proposed by Laws to apply an averaging window $(5 \times 5)$ on the images to get the energy measure for each pixel. Since we are already working with detected buildings and segmented shapes, we can speed up the processing by only applying the 25 kernels to pixels that are part of the detected buildings. We also propose to get a single averaged energy measure for each shape by summing and averaging the values for each pixel in the shape instead of using a rectangular window. Pixels on the edges of shapes are not used to avoid border effects.

Contrast normalization is obtained by dividing the results with the values of the L5L5 energy image. Orthogonal pairs (ex: A5B5 is orthogonal to B5A5) can be combined to reduce the number of features while the energy images from pure kernels (ex: A5A5) should be adjusted to keep equilibrium in the value range:

$$
\begin{gathered}
E 5 L 5_{\text {combined }}=E 5 L 5+L 5 E 5 \\
\ldots \\
E 5 E 5_{\text {adjusted }}=E 5 E 5 \times 2
\end{gathered}
$$

The final result is 14 textural features that can be used with the geometrical and scale features to evaluate building damage. In total, 27 features are used: intensity, scale, area, perimeter, 14 texture features, 4 geometric features and 5 scale features. To provide meaningful information to the classifier, we need to determine the changes that have occurred between the two image acquisition dates. To do so, we compute the feature differences for a building $m b_{b e f}$ with its matched postevent shape $m s_{a f t}$ :

$$
\begin{aligned}
& \Delta \operatorname{feat}_{S_{\text {diff }} w}\left(\operatorname{Match}\left(m b_{\text {befk }}, m s_{a f t f}\right)\right)=\mid \text { feat }_{m b_{\text {bef }} w}- \\
& \text { feat }_{m_{\text {aft }} w} \mid
\end{aligned}
$$

This gives us a new shape of differences $S_{\text {diff }}$ for each building from the pre-event image. Some of these shapes are used in the learning process while the others are used to assess the generalization performance of the classifier.

\section{F. Damage evaluation}

The last step in our proposed method is the damage estimation on the detected buildings. Three damage levels are considered for classification. The detailed process is presented in Fig. 5.

A multilayer backpropagation Perceptron neural network (MLP) is used for its simplicity and previous success in remote sensing applications. For this work, the set of shapes of differences $S_{\text {diff }}$ is randomly split into a learning set $S_{\text {diff_learn }}$ and a test set $S_{\text {diff_test }}$. The learning set is used to train the MLP with a subset for validation in order to optimize the parameters for the network (number of neurons, training epochs and learning rate). The test set is then classified into three damage classes using the trained network. In an operational setting, the selection of the learning set would be left to the operator who could also modify classes manually once the classification is done to remove false positives and to correct false negatives. This will lead to better final results while taking much less time than requiring that the operator manually assign each polygon and class. 


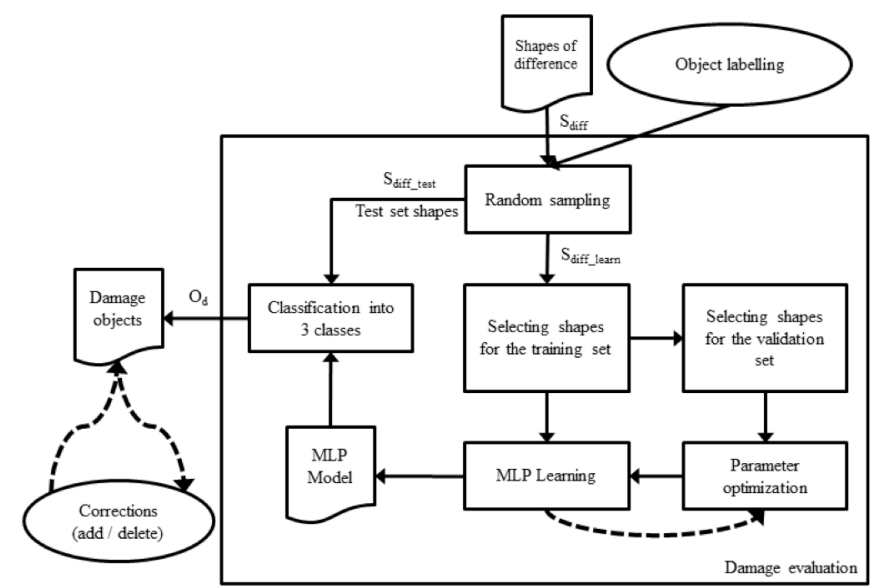

Fig. 5. Process diagram for the damage evaluation step (classification. [2 columns wide!!!]

\section{EXPERIMENTATION AND RESULTS}

\section{A. Data, hardware and software}

In order to test the proposed method, images acquired for the Haiti earthquake of January $12^{\text {nd }} 2010$ are analyzed. We use the Quickbird pre-event image acquired on February 22nd 2009 and the Quickbird post-event image acquired on January 15th 2010. These images were provided by MAGIC group of CSR from the University of Texas and Digital Globe. Both images were acquired in panchromatic and multispectral modes with spatial resolutions of $0.6 \mathrm{~m}$ and $2.4 \mathrm{~m}$ respectively but we only use the panchromatic bands. As a complement, we have also done preliminary tests with subsets of quickbird images of the case of the Boumerdes earthquake of 2003. These images were graciously provided by CNES and SERTIT.

The tests are executed on a personal computer running on an Intel Core ${ }^{\mathrm{TM}} 2 \mathrm{Duo}$ cpu at $2.33 \mathrm{GHz}$ with $4 \mathrm{~Gb}$ of memory. In order to assess our method, we use the "Atlas of building damage assessment Haiti earthquake 12 January 2010" [26] produced by the United Nations Institute for Training and Research (UNITAR) Operational Satellite Applications Programme (UNOSAT), the European Commission (EC) Joint Research Center (JRC) and the World Bank (WB) as ground truth since it is the most complete and extensive study available. Both the vector layer and the raster images were put in WGS 84 / UTM zone 18N coordinates for superimposition. For the Boumerdes set, we use the vector data layers provided by the relevant organizations as ground truth.

The algorithms are implemented using the Orfeo Toolbox (OTB), an open-source remote-sensing library developed in part by the CNES [27]. Fig. 6 below shows the region of Portau-Prince near the presidential palace. Two subsets are extracted from this region. Each subset is $800 \times 600$ pixels and is shown bounded by a white box.

In Fig. 7, we show subset BOU1 before and after the disaster. Note that multiple buildings had soft story collapses with scarce apparent rubble.

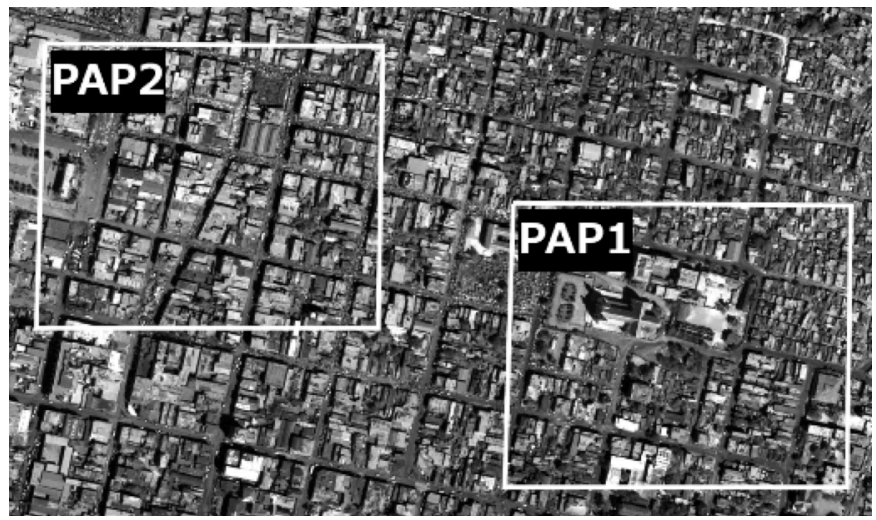

Fig. 6. Port-au-Prince region where two subsets are used for testing the proposed method.

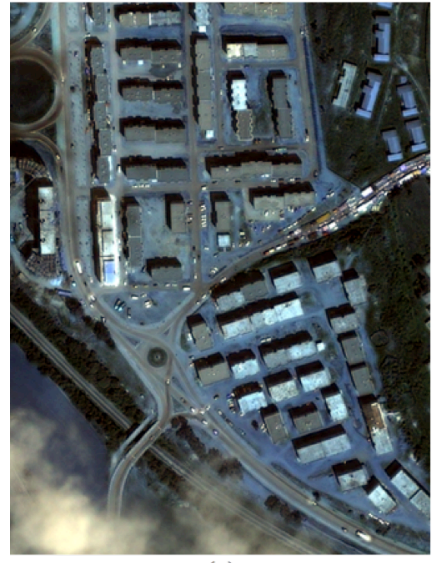

(a)

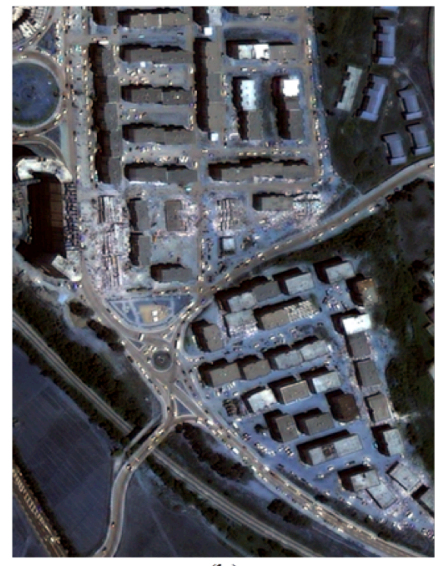

(b)
Fig. 7. Boumerdes test set 1(BOU1) before (a) and after (b) the event.

\section{B. Parameters and their optimization}

The various steps of the proposed method require setting different parameters. Although there are few parameters, it is important to set them using a priori information on the scene, e.g. the buildings' shapes and sizes and the required precision. For our first subset which is composed of the streets surrounding the Cathedral of Our Lady of the Assumption (PAP1 zone), we set the minimal building surface as 75 pixels, the maximal building surface as 3575 pixels and a value of 1 for the $\lambda$ and $\gamma_{s}$ parameters for blur amount and shape regularity respectively. The FLST transformation is also limited to outputting shapes with an area greater than 75 pixels to reduce the number of shapes to consider. We use a MLP neural network with 14 neurons in the hidden layer and 3 neurons for output. The hidden neurons use a hyperbolic tangent transfer function and the output neurons a sigmoid transfer function. The learning rate is set to 0.15 and the maximum number of epochs is set to 800 . In order to obtain these parameters, we have generated a set of classifiers with varying parameters and have tested them with 100 sets of training data from our pool of damage-labeled buildings. The results are presented in Fig. 8. For each parameter tested, the other parameters were set to the optimal value found.

We labeled 533 building shapes into the three damage classes according to [4] by using the vector layer from the 


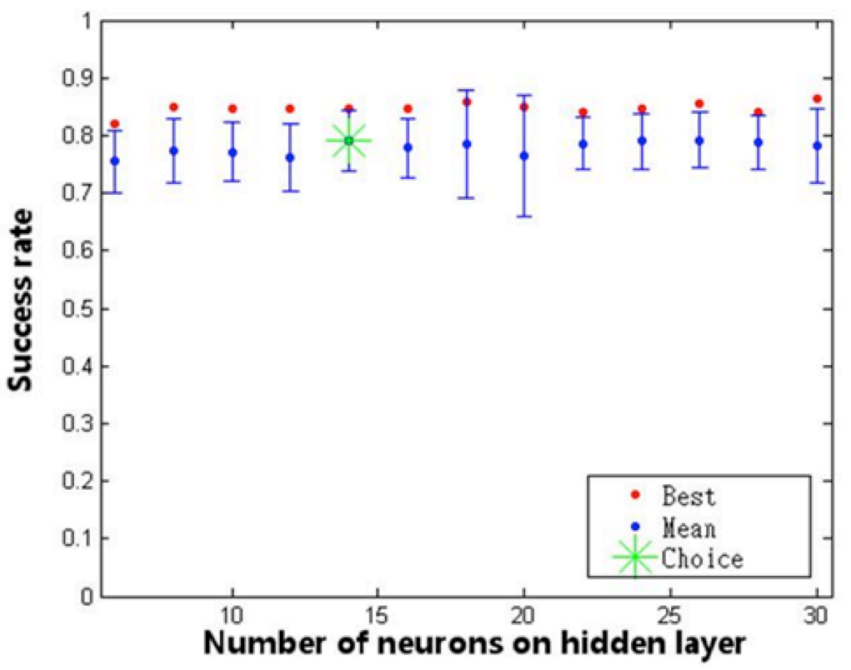

(a)

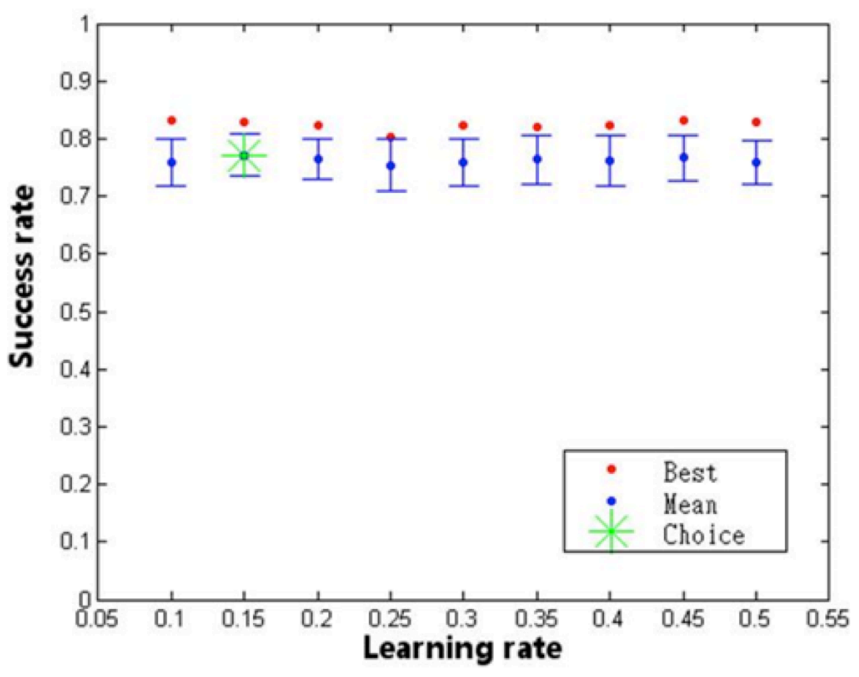

(b)

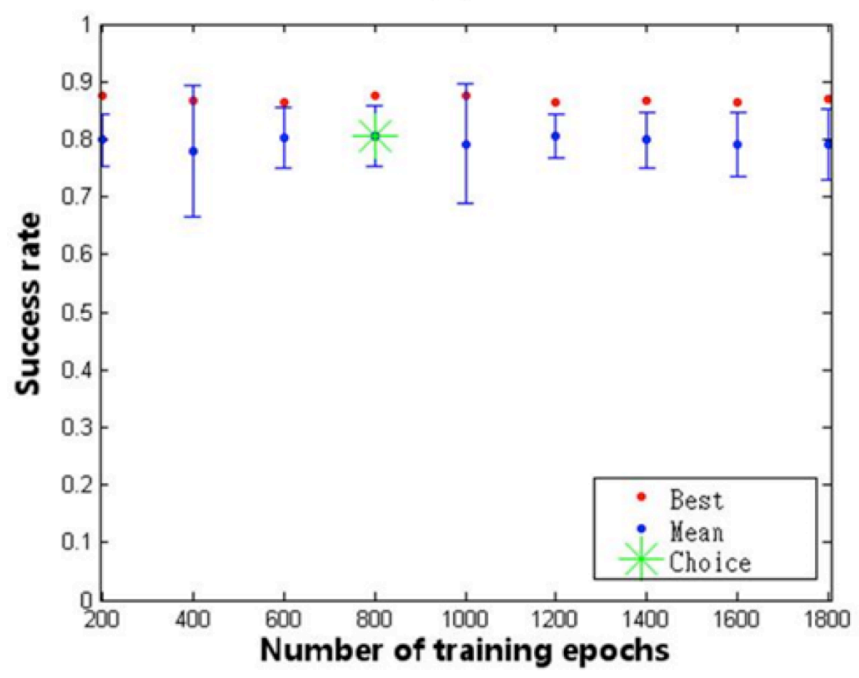

(c)

Fig. 8. Validation runs for MLP parameters selection. Number of neurons (a), learning rate (b) and number of training epochs (c).

Atlas of building damage assessment. We used 200 samples for training the network, 100 for validation and 233 for testing for 100 iterations. For each iteration, the samples for the three sets are randomly selected (with no overlap).

For our second subset (PAP2 zone) which is a region a few blocks to the West of the first one, we use the same extraction parameters. These parameters yields 415 building shapes that we also labeled again by using the vector layer from the Atlas of building damage assessment. Of those buildings, 115 are used for testing.

For our third and fourth subsets (BOU1 and BOU2 respectively), we use 35 shapes out of 74 for BOU1 and 35 shapes out of 78 for BOU2. These regions have a much lower density than PAP1 and PAP2 and most buildings are large apartment complexes.

\section{Shape matching and damage evaluation}

The extracted shapes are matched to buildings from the preevent image leading to pairs of shapes. Features difference is calculated on these pairs to form a difference vector used for damage classification.

Three damage classes are considered: no damage to low damage (ok), damage (dmg.) and heavy damage or destruction (dest.). These classes correspond to EMS-98 grades 0 to 3, grade 4 and grade 5 respectively [28]. As stated, Law's textural features are combined with the previous features used for shape matching. The visual results are presented in Fig. 9. Table I shows the confusion matrix for the PAP1 test set. The columns show World Bank, UNOSAT, Joint Research Center field survey and visual analysis results [26] and rows are results from our method. Results are good with high producer and user accuracies and a global accuracy of $84.1 \%$. Interestingly, the middle class is never confused with the destroyed class while the intact and destroyed classes both have a comparable number of buildings classified falsely as the other class. This can, in part, be explained by the change in viewing angle between image acquisitions and by the changes not related to the earthquake (such as new constructions, demolished or renovated buildings). We also test the trained network on the PAP2 test set and obtain similar results with a global accuracy of $84.3 \%$. Detailed results are included in table II. We note some major differences between the user and producer accuracies from one set to the other. It is important to note that the PAP2 region contains generally bigger buildings than the PAP1 region leading to more precise texture and scale profile measures and thus better discrimination of damage which probably account for these differences. The visual results for PAP2 are presented in Fig. 10. As for BOU1 and BOU2 test sets, the results are presented in Table III.

We can see that mean accuracy is greatly affected by the smaller number of learning samples. Another difficulty here is that the ground truth identifies segments of buildings as being destroyed while other segments are deemed damaged or unaffected. The segmentation scheme is thus not adapted to the ground truth classification method for the large buildings in this set since buildings would need to be further segmented. 


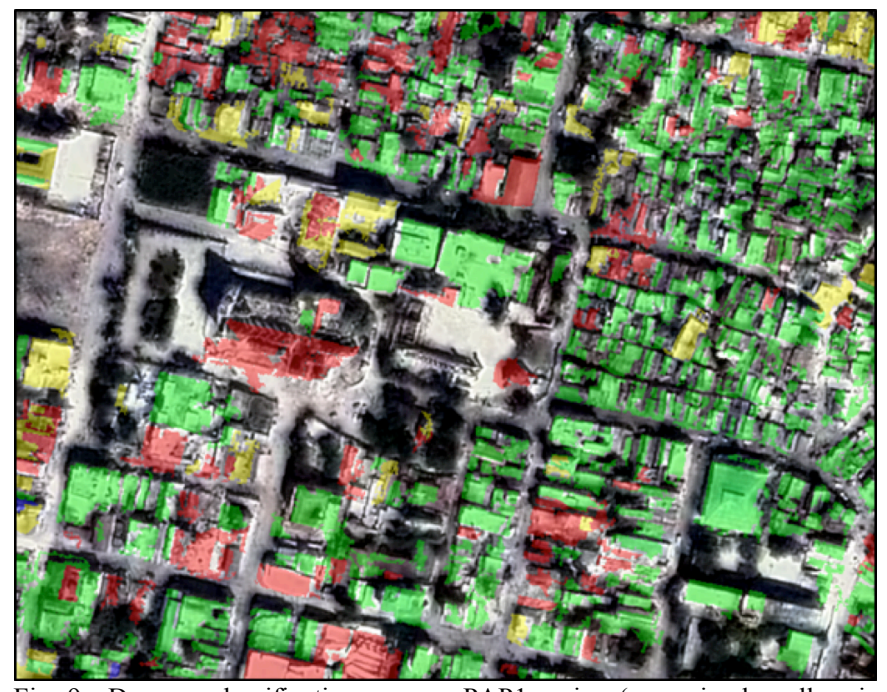

Fig. 9. Damage classification map on PAP1 region (green is ok, yellow is damaged and red is destroyed).

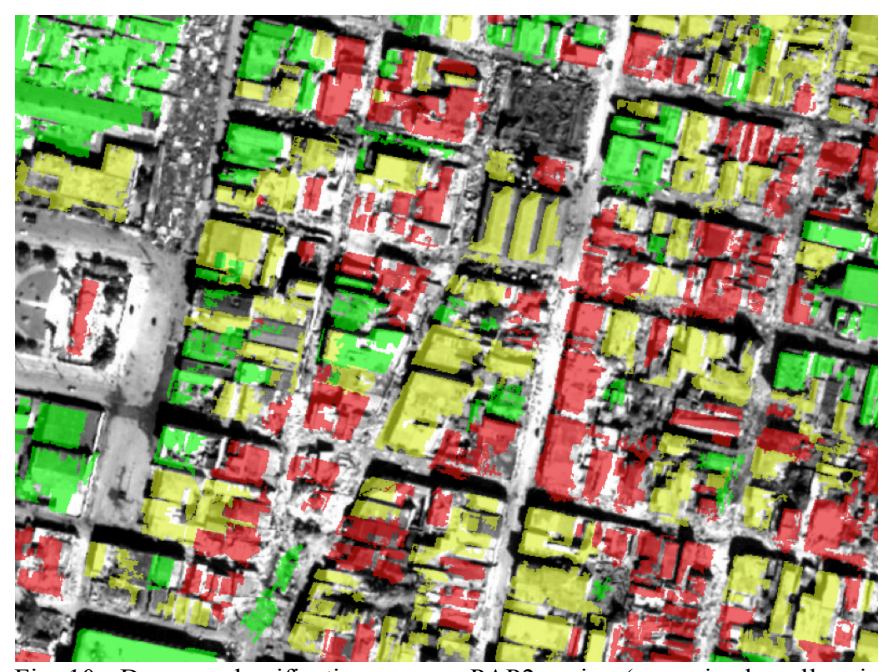

Fig. 10. Damage classification map on PAP2 region (green is ok, yellow is damaged and red is destroyed).

TABLE I

CONFUSION MATRIX FOR DAMAGE CLASSIFICATION OF REGION PAP1

Reference

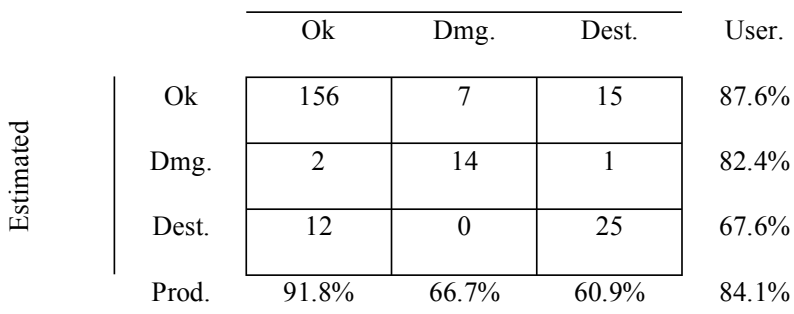

TABLE II

CONFUSION MATRIX FOR DAMAGE CLASSIFICATION OF REGION PAP2 Reference

\begin{tabular}{|c|c|c|c|c|}
\hline \multirow{5}{*}{ 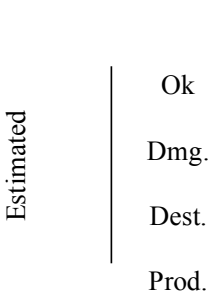 } & $\mathrm{Ok}$ & Dmg. & Dest. & User. \\
\hline & 26 & 1 & 2 & $89.7 \%$ \\
\hline & 3 & 34 & 4 & $82.9 \%$ \\
\hline & 3 & 5 & 37 & $82.2 \%$ \\
\hline & $81.2^{\circ}$ & $85 \%$ & $86 \%$ & $84.3 \%$ \\
\hline
\end{tabular}

TABLE III TEST RESULTS FOR ALL DATASETS

\begin{tabular}{cccc}
\hline \multicolumn{3}{c}{ TEST RESULTS FOR ALL DATASETS } \\
\hline & $\begin{array}{c}\text { Overall } \\
\text { accuracy } \\
(\%)\end{array}$ & $\begin{array}{c}\text { Mean } \\
\text { overall } \\
\text { accuracy } \\
(\%)\end{array}$ & $\begin{array}{c}\text { Std. } \\
\text { deviation }\end{array}$ \\
\hline PAP1 & 84 & 78.7 & 2.8 \\
PAP2 & 75 & 71.2 & 5.1 \\
BOU1 & 84 & 70.1 & 5.7 \\
BOU2 & 72 & 61.4 & 6.1 \\
\hline \hline
\end{tabular}

\section{Comparison with other methods}

Since our method (geometry, scale and texture or GST) only requires a single panchromatic pre-disaster image and a single post-disaster image, it should be compared to a method that has similar prerequisites. For this comparison, we chose the supervised correlation classification scheme proposed in $[7,29]$. This method computes pixel to pixel correlation on the intensity values of the pre- and post-event images as well as on the edge maps derived from these images. Only the pixels inside predefined building footprints are considered to lighten the processing load. All the results are presented in Table IV for PAP1 and in Table $\mathrm{V}$ for BOU1. We used the same training sets for both methods as well as the validation and test sets. The processing time does not include the labelling of the training data as it is the same for both methods since the same shapes are used. For both disaster events, the proposed method gives better overall accuracy while requiring less processing time. 
TABLE IV

COMPARISON OF THE PROPOSED METHOD (GST - GEOMETRY, SCALE AND TEXTURE) AND THE CORRELATION CLASSIFICATION METHOD FOR PAP1

\begin{tabular}{ccccccccc}
\hline \hline & \multicolumn{1}{c}{$\begin{array}{c}\text { Overall } \\
\text { accuracy } \\
(\%)\end{array}$} & $\begin{array}{c}\text { Prod. Prod. Prod. } \\
\text { for } \\
\text { ok } \\
(\%)\end{array}$ & $\begin{array}{c}\text { for } \\
\text { dm. } \\
(\%)\end{array}$ & $\begin{array}{c}\text { for } \\
(\%)\end{array}$ & $\begin{array}{c}\text { Mean } \\
\text { overall } \\
\text { accuracy } \\
(\%)\end{array}$ & $\begin{array}{c}\text { Std. } \\
\text { deviation }\end{array}$ & $\begin{array}{c}\text { Processing } \\
\text { time (s) }\end{array}$ \\
\hline GST & 84 & 92 & 67 & 61 & 0.6 & 78.7 & 2.8 & 345 \\
Corr. & 75 & 98 & 5 & 19 & 0.18 & 71.2 & 5.1 & 560 \\
\hline \hline
\end{tabular}

TABLE V

COMPARISON OF THE PROPOSED METHOD (GST - GEOMETRY, SCALE AND TEXTURE) AND THE CORRELATION CLASSIFICATION METHOD FOR BOU1

\begin{tabular}{ccccccccc}
\hline \hline & \multicolumn{1}{c}{$\begin{array}{c}\text { Overall } \\
\text { accuracy } \\
(\%)\end{array}$} & $\begin{array}{c}\text { Prod. Prod. Prod. } \\
\text { for } \\
\text { ok } \\
(\%)\end{array}$ & $\begin{array}{c}\text { for } \\
\text { dm. }\end{array}$ & $\begin{array}{c}\text { for } \\
\text { dest. } \\
(\%)\end{array}$ & $\begin{array}{c}\text { Kean } \\
\text { overall } \\
\text { accuracy } \\
(\%)\end{array}$ & $\begin{array}{c}\text { Std. } \\
\text { deviation }\end{array}$ & $\begin{array}{c}\text { Processing } \\
\text { time (s) }\end{array}$ \\
\hline GST & 84 & 96 & 60 & 73 & 0.72 & 70.1 & 5.7 & 122 \\
Corr. & 64 & 100 & 40 & 0 & 0.39 & 56.5 & 4.8 & 213 \\
\hline \hline
\end{tabular}

Calculating the class-weighted overall accuracy highlights even more the gap between our method and the correlation method with values of $73.1 \%$ and $40.6 \%$ respectively for PAP1. This major difference is explained by the proportion of buildings in each class. The no damage to low damage class effectively accounts for almost $3 / 4$ of all buildings so a high accuracy for this class will greatly contribute to the global accuracy.

We also compare our method to the visual classification done by the SERTIT group for the Charter activation \#287 (Haiti 2010 Earthquake) [30]. The accuracy of both methods is calculated according to classification differences compared to the ground truth from the Atlas of building damage assessment. In this case, two important modifications are made. First, we only take into account the buildings contained in the PAP1 region we defined instead of the whole city. Second, since the damage maps created from visual inspection is binary (damage or no damage), we merge classes for grade 4 and for grade 5 into a "damage" class to compare the two methods. The results are presented in Table VI.

TABLE VI

COMPARISON OF THE PROPOSED METHOD AND THE VISUAL INTERPRETATION METHOD

\begin{tabular}{|c|c|c|c|c|}
\hline & $\begin{array}{c}\text { Overall } \\
\text { accuracy } \\
(\%)\end{array}$ & $\begin{array}{c}\text { Prod. } \\
\text { for } \\
\text { ok } \\
(\%) \\
\end{array}$ & $\begin{array}{c}\text { Prod. } \\
\text { for } \\
\text { dmg. } \\
(\%)\end{array}$ & ב Kappa \\
\hline GST & 84 & 92 & 65 & 0.59 \\
\hline Visu. & 76 & 79 & 63 & 0.35 \\
\hline
\end{tabular}

Even if we only consider that the visual damage assessment method assigns a damage level without extracting the complete footprint of the building, the expert will require more than a few seconds for each building. This leads to an interpretation time that cannot be under 20 minutes for a region of the size and building density similar to PAP1.

As can be seen here, our method outperforms both visual inspection methods and current optical based automated methods making it a viable choice for fast damage evaluation when a limited amount of images are available. Shortcomings include the need for an expert operator for learning set selection and that an archive image is required, but this will be less of a problem with the growing archives and their availability through the Charter.

\section{CONCLUSION}

In conclusion, we have presented a method based on semiautomated evaluation of building damages in disaster images. The method we propose is ideal for a number of reasons. First, it is faster than most existing damage evaluation methods while requiring only two panchromatic images instead of multispectral images or multiple images from different viewing angles, as required for the pictometry method. Second, we have demonstrated that its accuracy is sufficient to attain better results compared with traditional visual inspection. Third, it requires few parameters that are closely tied to physical properties of the scene like buildings sizes and shapes. These properties make our method ideal for use in the frame of the Charter. Finally, by using a desktop with a four cores CPU and considering that each step is linearly scalable, the whole downtown region of Port-au-Prince could be processed in two to three hours.

For future work, we plan to evaluate the error propagation impact of using a sequential framework with two classifiers. A multistage optimization scheme like the one proposed in [31] will be considered. Future investigation is also required to pinpoint key features for the discrimination of soft-story collapses which are a great source of omitted damage in automated and manual inspection of remotely sensed data. Possible solutions include using sensor view angle, sun elevation information and building shadows from before and after disaster images to determine changes in a building's height which can give better discrimination for soft story collapses. Optimization of the feature extraction and shape matching algorithms is also considered to accelerate the process. We plan to test our method on another dataset (cases of earthquakes in Bam, Iran) and do complete tests with the Haiti and Boumerdes datasets (such as using a single model to classify all regions and retraining an existing model with a few samples from a new event).

\section{ACKNOWLEDGMENT}

D. Dubois wishes to thank Pascal Monasse for taking the time on multiple occasions to answer his queries on the workings of the FLST. The authors also wish to thank the 
MAGIC group of CSR from the University of Texas and Digital Globe for providing some of the raw data used for this research.

\section{REFERENCES}

[1] J. L. Bessis, J. Béquignon, and A. Mahmood, "The International Charter "Space and Major Disasters" initiative," Acta Astronautica, vol. 54, pp. 183-190, 2004

[2] M. Gerke and N. Kerle, "Automatic Structural Seismic Damage Assessment with Airborne Oblique Pictometry Imagery," Photogrammetric Engineering and Remote Sensing, vol. 77, pp. 885898, September 2011.

[3] E. Hussain, S. Ural, K. Kim, C.-S. Fu, and J. Shan, "Building Extraction and Rubble Mapping for City Port-au-Prince Post-2010 Earthquake with GeoEye-1 Imagery and Lidar Data," Photogrammetric Engineering and Remote Sensing, vol. 77, pp. 1011-1023, October 2011.

[4] C. Corbane, K. Saito, L. Dell'Oro, E. Bjorgo, S. P. D. Gill, B. E. Piard, et al., "A Comprehensive Analysis of Building Damage in the 12 January 2010 Mw7 Haiti Earthquake Using High-Resolution Satelliteand Aerial Imagery," Photogrammetric Engineering and Remote Sensing, vol. 77, pp. 997-1009, October 2011.

[5] F. Dell'Acqua and D. A. Polli, "Post-event Only VHR Radar Satellite Data for Automated Damage Assessment: A Study on COSMO/SkyMed and the 2010 Haiti Earthquake," Photogrammetric Engineering and Remote Sensing, vol. 77, pp. 1037-1043, October 2011.

[6] S. Voigt, T. Schneiderhan, A. Twele, M. Gähler, E. Stein, and H. Mehl, "Rapid Damage Assessment and Situation Mapping: Learning from the 2010 Haiti Earthquake," Photogrammetric Engineering and Remote Sensing, vol. 77, pp. 923-931, September 2011.

[7] A.-L. Chesnel, R. Binet, and L. Wald, "Urban damage assessment using multimodal QuickBird images and ancillary data: the Bam and the Boumerdes earthquakes," in 6th International Workshop on Remote Sensing for Disaster Management Applications, ed, 2008.

[8] P. Li, H. Xu, and J. Guo, "Urban building damage detection from very high resolution imagery using OCSVM and spatial features," International Journal of Remote Sensing, vol. 31, pp. 3393-3409, 2010.

[9] S. Klonus, D. Tomowski, M. Ehlers, P. Reinartz, and U. Michel, "Combined Edge Segment Texture Analysis for the Detection of Damaged Buildings in Crisis Areas," IEEE Journal of Selected Topics in Applied Earth Observations and Remote Sensing, vol. 5, pp. 1118-1128, 2012

[10] T. Balz and M. Liao, "Building-damage detection using post-seismic high-resolution SAR satellite data," International Journal of Remote Sensing, vol. 31, pp. 3369-3391, 2010.

[11] M. Matsuoka and N. Nojima, "Building Damage Estimation by Integration of Seismic Intensity Information and Satellite L-band SAR Imagery," Remote Sensing, vol. 2, pp. 2111-2126, 2010.

[12] R. Cossu, F. Dell'Acqua, D. A. Polli, and G. Rogolino, "SAR-Based Seismic Damage Assessment in Urban Areas: Scaling Down Resolution, Scaling Up Computational Performance," IEEE Journal of Selected Topics in Applied Earth Observations and Remote Sensing, vol. 5, pp. 1110-1117, 2012

[13] M. Chini, N. Pierdicca, and W. J. Emery, "Exploiting SAR and VHR optical images to quantify damage caused by the 2003 bam earthquake," IEEE Transactions on Geoscience and Remote Sensing, vol. 47, pp. 145$152,2009$.

[14] D. Dubois and R. Lepage, "Meeting Remote Sensing Requirements for Faster Disaster Response," presented at the International Geoscience and Remote Sensing Symposium, 2012.

[15] R. Saint-Jean, "The role of the ECO in the context of the international charter on space and major disasters," in Proceedings of the Geoscience and Remote Sensing Symposium. vol. 6, ed Seoul, Korea: IEEE, 2005, pp. 4370-4372.

[16] P. Monasse and F. Guichard, "Fast computation of a contrast-invariant image representation," IEEE Transactions on Image Processing, vol. 9, pp. $860-872,2000$.

[17] J. A. Benediktsson, M. Pesaresi, and K. Amason, "Classification and feature extraction for remote sensing images from urban areas based on morphological transformations," IEEE Transactions on Geoscience and Remote Sensing, vol. 41, pp. 1940-1949, 2003.

[18] T. Brox and J. Weickert, "A TV flow based local scale measure for texture discrimination," Computer Vision-ECCV, pp. 578-590, 2004.
[19] T. Lindeberg, "Feature detection with automatic scale selection," International Journal of Computer Vision, vol. 30, pp. 79-116, 1998.

[20] A. Winter, H. Maitre, N. Cambou, and E. Legrand, "Entropy and multiscale analysis: a new feature extraction algorithm for aerial images," in IEEE International Conference on Acoustics, Speech, and Signal Processing, Los Alamitos, CA, USA, 1997, pp. 2765-8.

[21] B. Luo, J. F. Aujol, Y. Gousseau, S. Ladjal, and H. Maitre, "ResolutionIndependent Characteristic Scale Dedicated to Satellite Images," IEEE Transactions on Image Processing, vol. 16, pp. 2503-2514, 2007.

[22] B. Luo, J.-F. Aujol, and Y. Gousseau, "Local scale measure from the topographic map and application to remote sensing images," in SIAM Journal on Multiscale Modeling and Simulation vol. 8, ed, 2009, pp. 129.

[23] D. Dubois and R. Lepage, "Scale profile as feature for quick satellite image object-based classification," in SPIE Defense, Security, and Sensing, 2013, pp. 87431Z-87431Z-9.

[24] V. Caselles and P. Monasse, Geometric Description of Images as Topographic Maps. Berlin (Allemagne): Springer-Verlag, 2009.

[25] K. I. Laws, "Textured Image Segmentation," PhD dissertation, University of Southern California, Los Angeles, 1980.

[26] UNITAR, Operational Satellite Applications Programme, European Commission, Joint Research Centre, and The World Bank, "Atlas of building damage assessment: Haiti earthquake 12 January 2010," UNITAR 2010.

[27] E. Christophe and J. Inglada, "Open Source Remote Sensing: Increasing the Usability of Cutting-Edge Algorithms," IEEE Geoscience and Remote Sensing Society Newsletter, pp. 9-15, Mach 2009.

[28] G. Grünthal, European Macroseismic Scale 1998 (EMS-98). Luxembourg, 1998.

[29] A.-L. Chesnel, "Quantification de dégâts sur le bâti liés aux catastrophes majeures par images satellite multimodales très haute résolution," $\mathrm{PhD}$ dissertation, CEP - Centre Énergétique et Procédés, Ecole nationale supérieure des Mines de Paris, Paris, 2008.

[30] International Charter, Space and Major Disasters. (2010, 27 octobre 2010). Earthquake in Haiti [Online]. Available: http://www.disasterscharter.org/web/charter/activation_details?p_r_p_14 15474252 assetId $=$ ACT -287

[31] T. Landgrebe, P. Paclík, D. M. Tax, and R. P. Duin, "Optimising twostage recognition systems," in Multiple Classifier Systems, ed: Springer, 2005 , pp. 206-215.

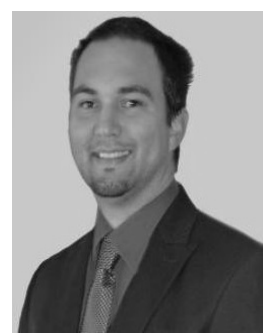

David Dubois (GSM'09). This author became a Graduate Student Member (GSM) of IEEE in 2009. The author obtained his bachelor's degree in electrical engineering and his master's degree in automated process engineering and his Ph.D. from École de technologie supérieure, Montréal, Québec, Canada in 2007, 2010 and 2014 respectively. The author is currently a postdoctoral fellow at École de technologie supérieure. His major field of study is automated computer vision processes.

He has been a Teaching Assistant for computer vision and artificial intelligence related courses since 2007 at École de technologie supérieure. His current research interests include computer vision, artificial intelligence, process optimization and remote sensing.

Dr. Dubois is also a member of his province's engineering professional association as well as a student member of SPIE. He was Chair of the IEEE Student Branch of the École de technologie supérieure university for more than a year.

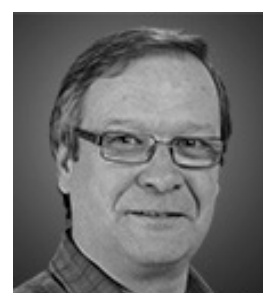

Richard Lepage (M'13) received the degree in electrical engineering in 1974, the M.S. degree in signal processing in 1982, and the Ph.D. degree for work on 
applications of artificial neural networks to computer vision, in 1993, all from from Laval University, Quebec, P.Q., Canada.

He is currently a Professor in the Department of Automated Production, École de Technologie Supérieure, Université du Québec, Montréal, P.Q. From 1976 to 1989, he was with the Defense Research Center, Quebec, Canada. His research interests include neural networks, computer vision, 3-D vision, automated inspection systems, and the use of artificial intelligence techniques in remote sensing. 